\title{
Discrepancia en la evaluación de la calidad de vida relacionada con la salud entre niños y padres a través del cuestionario EQ-5D-Y

\author{
Parent-child discrepancy in the assessment of health-related \\ quality of life using the EQ-5D-Y questionnaire
}

\author{
Dr. Miguel Á. Perez Sousa ${ }^{a}$, Dr. Pedro R. Olivares Sánchez-Toledo ${ }^{b}$ \\ y Cat. Narcis Gusi Fuerte
}

\section{RESUMEN}

Introducción. La evaluación de la calidad de vida relacionada con la salud (CVRS) permite detectar cambios en el tiempo en la salud de pacientes y posibilita realizarunanálisis decoste-efectividad de tratamientos. Enniños con característicasespeciales de salud que no pueden autoevaluarse, existe la posibilidad de evaluar su CVRS a través de padres o cuidadores. Hasta la fecha, no se ha analizado la discrepancia en la evaluación de la CVRS a través del cuestionario EQ-5D-Y entre niños con parálisis cerebral (PC) y sus padres. El objetivo del presente estudio fue analizar el grado de concordancia en la evaluación de la CVRS a través del cuestionario EQ-5D-Y y su versión Proxy entre niños con PC y sus padres o cuidadores.

Población y métodos. Participaron, en el estudio, niños y adolescentes con PC, así como sus padres y madres, procedentes de un centro de educación especial dela región de Extremadura (España). Se utilizó el cuestionario EQ-5D-Y para los niños y el EQ-5D-Y Proxy para los padres. Las entrevistas fueron llevabas a cabo duranteel primer trimestre de 2015. Se analizó la concordancia en las respuestas medianteelniveldeacuerdoconkappa deCohen para las 5 dimensiones que componenel EQ-5D-Y y el coeficiente de correlación intraclase para la escala visual analógica.

a. Facultad de Ciencias del Deporte, Universidad de Extremadura.

b. Instituto de Investigación en Actividad Física y Salud, Universidad Autónoma de Chile, Talca, Chile. olivares.pedro@ gmail.com.

Correspondencia: Dr. Miguel Á. Pérez Sousa: maperezsousa@unex.es.

Financiamiento:

Ninguno.

Conflicto de intereses: Ninguno que declarar.

Recibido: 15-12-2016 Aceptado: 19-5-2017
Cómo citar: Perez Sousa MA, Olivarez Sánchez-Toledo PR, Gusi Fuerte N. Discrepancia en la evaluación de calidad de vida relacionada con salud entre niños y padres a travéz del cuestionario EQ-5D-Y. Arch Argent Pediatr 2017;115(6):541-546.

\section{INTRODUCCIÓN}

La evaluación de la calidad de vida relacionada con la salud (CVRS), entendiendo esta como un término multidisciplinar que complementa los resultados médicos, fisiológicos y bioquímicos, ${ }^{1}$ ha crecido en la última década y ha aumentado la disponibilidad de cuestionarios genéricos, como PedsQL, ${ }^{2}$ Kidscreen ${ }^{3}$ y Euroqol 5D. ${ }^{4}$ Si bien es cierto que estos cuestionarios para la autoevaluación de la CVRS han sido validados para diferentes poblaciones con características especiales de salud, existen otras que, por sus diferentes particularidades, ya sean de salud o socioculturales, son incapaces de autoevaluarse, lo que genera la aparición de la figura de un evaluador externo con relación directa con el paciente. Por este motivo, surgen versiones de cuestionarios de evaluación de la CVRS capaces de evaluarla a través de un proxy o persona sustituta, que son, comúnmente, padres, tutores o agentes de salud.

En los últimos años, los principales instrumentos de evaluación de la CVRS, como EQ-5D-Y, Kidscreen y PedsQL, han desarrollado su versión para proxy y han demostrado su fiabilidad y validez. ${ }^{5-9}$

Siendo nuestro objeto de estudio la CVRS en niños y adolescentes, se nos abre un rango de posibilidades para evaluarla, ya que el uso de cuestionarios proxy no solo se aplica a niños y adolescentes que padecen enfermedades limitantes, sino también a niños que tienen dificultad de 
comprensión lectora o pertenecen a un estatus socioeconómico bajo. En este sentido, los padres son esenciales en la evaluación de la CVRS de sus hijos ${ }^{10}$ y contribuyen de manera crucial en la decisión del tratamiento para aplicar por parte de médicos y especialistas ${ }^{11,12}$ y en la detección de cambios en el tiempo en la CVRS del niño. ${ }^{13}$ No obstante, la evaluación por parte del proxy nunca puede suplir la del niño/paciente si este la puede realizar correctamente, ya que se obtiene una evaluación más precisa en dimensiones psicosociales. ${ }^{14,15}$

Se ha descrito, en diversos estudios, un mayor o menor grado de acuerdo o desacuerdo entre niño/paciente y proxy tanto en población sana como en población con características especiales de salud. ${ }^{14-19}$ Respecto a niños con problemas motores, como parálisis cerebral (PC), se ha encontrado un alto grado de desacuerdo entre niños y padres, y se ha evaluado una pobre CVRS de sus hijos, ${ }^{17}$ al igual que sucede en niños con distrofia muscular. ${ }^{20}$ Por tanto, se encuentra mucha ambigüedad en el nivel de acuerdo entre el proxy y los niños en población sana y con características especiales de salud, quizás, por la dificultad que entraña en sí misma o por lo extenso de los cuestionarios empleados.

En esta línea, el cuestionario EQ-5D-Y Proxy adaptado y validado al español ${ }^{6}$ presenta las mismas características que la versión Proxy para adultos y mayores. Es corto, fácil de administrar y proporciona resultados de diferentes dimensiones de la salud, así como un valor de índice que se puede utilizar para evaluar el estado de salud. También es útil para el análisis de evaluación económica de servicios sanitarios. Sin embargo, no se ha investigado el nivel de acuerdo/desacuerdo entre sujeto/paciente y su proxy en niños con PC.

El objetivo del presente estudio fue analizar el grado de concordancia entre niños con PC y población sana, así como padres y madres, empleando el cuestionario EQ-5D-Y y su versión Proxy. La hipótesis planteada fue que existía un alto desacuerdo en la evaluación de la CVRS entre padres e hijos con PC.

\section{POBLACIÓN Y MÉTODOS Participantes}

Para la selección de la muestra, se acudió a tres centros de educación especial de la comunidad de Extremadura. Luego de presentar el protocolo de investigación a la dirección del centro y consejo de padres de alumnos, aceptó participar en el estudio, tras la firma del consentimiento, un centro de educación especial para personas con PC.
En el estudio, que fue realizado durante el primer trimestre de 2015, participaron niños de entre 6 y 17 años con PC procedentes de un centro especializado, clasificados según su capacidad funcional en grado uno (sin limitación de actividad) y grado dos (con ligera o moderada limitación de la actividad), así como sus respectivos padres y madres. Para la participación, se exigía la firma del consentimiento informado tanto para niños como para padres.

Los sujetos completaron la versión autorreportada para niños y la versión Proxy para padres del cuestionario EQ-5D-Y.

Los criterios de inclusión que debían cumplir los participantes fueron los siguientes:

1. Padecer PC en grado 1 (sin limitación alguna de la actividad) o 2 (con ligera o moderada limitación de la actividad), clasificados por el equipo médico-terapeuta del centro en función del grado de capacidad funcional.

2. Poseer la suficiente capacidad de comprensión lectora para entender los ítems y responder.

3. Personarse el día de la prueba.

4. Firmar el consentimiento informado por parte del sujeto y de los padres o tutores.

Estos criterios de admisión fueron aplicados por el equipo de terapeutas del centro.

Las entrevistas se llevaron a cabo en aulas destinadas para tal fin en cada centro y estas fueron realizadas por un técnico con experiencia en este tipo de estudios. El cuestionario fue aplicado mediante entrevistas individuales y el tiempo aproximado para completarlo fue de 8 minutos.

Las entrevistas a los padres se realizaron, por una parte, a las madres y, por otra, a los padres en aulas separadas con la citación previa en cada centro correspondiente. El estudio fue aprobado por el Comité de Bioética de la Universidad de Extremadura y se realizó siguiendo las Pautas éticas internacionales para la investigación biomédica en seres humanos de Ginebra.

\section{Instrumentos}

EQ-5D-Y y versión Proxy: La versión española del cuestionario EQ-5D-Y $Y^{21}$ y su versión Proxy ${ }^{6}$ comprenden una sección descriptiva de cinco dimensiones con 3 niveles de respuesta ("no tengo problemas", "tengo algunos problemas" y "tengo muchos problemas"). Las dimensiones de las que se compone el cuestionario son movilidad, autocuidado, actividades habituales, dolor y malestar, y ansiedad y depresión. También incluye una escala visual analógica (EVA), en la que el sujeto 
realiza una evaluación global de su estado de salud en una escala de 0 a 100: 0 representa el peor estado de salud, y 100, el mejor estado de salud.

La versión Proxy, completada por los padres, que responden sobre la CVRS de sus hijos, posee las mismas características que la versión autocompletada.

Este cuestionario evalúa la CVRS atendiendo a cinco aspectos clave en la calidad de vida de las personas: la capacidad para caminar, las actividades relacionadas con el autocuidado (lavarse o vestirse solo), la realización de las actividades habituales de la vida diaria (ir al colegio, jugar o actividades con la familia y amigos), el dolor o malestar que pueda padecer $y$, por último, la ansiedad o depresión. Finalmente, el cuestionario ofrece la frecuencia de problemas reportados por el propio sujeto o, en el caso de la versión para proxy, por un evaluador externo con relación directa con el niño/paciente.

El EQ-5D-Y y la versión Proxy han sido previamente testados, a través del cuestionario para la evaluación de instrumentos de medida de resultados percibidos por los pacientes (EMPRO), por el equipo de evaluadores del grupo de investigación, y han demostrado una puntuación alta en las áreas de fiabilidad, validez, interpretabilidad, facilidad de uso, alternativas de aplicación y adaptación cultural, y lingüística. Además, el uso de este cuestionario está muy extendido internacionalmente en diferentes poblaciones con características especiales de salud, ${ }^{22-25}$ cuyas propiedades métricas han sido descritas en su versión española para España, ${ }^{6}$ así como su comprensibilidad y adaptación cultural en población general. ${ }^{7}$ En este sentido, se ha realizado previamente un estudio piloto para evaluar la comprensibilidad mediante métodos de indagación y parafraseo en la población objetivo y se ha presentado una óptima comprensibilidad.

También se incluyeron preguntas sobre la frecuencia de problemas de salud durante los últimos 6 meses, tales como dolor de cabeza, estómago, espalda, bajo ánimo, irritabilidad, nerviosismo o dificultades para dormir, y preguntas sociodemográficas, tales como sexo, edad, país de procedencia o idiomas.

\section{Análisis}

Se realizó un cálculo del tamaño muestral para un nivel de significación de 0,05 y un nivel de confianza de $90 \%$.

El análisis estadístico se realizó con el programa SPSS 21.0. Para observar la normalidad y distribución de los datos, se aplicó media, desviación estándar y frecuencias. El nivel de concordancia en la respuesta de las cinco dimensiones del EQ-5D-Y y Proxy se analizó mediante kappa de Cohen siguiendo los criterios de Landis y Koch, ${ }^{26}$ y se estableció un nivel de acuerdo para kappa nulo: <0; 0-0,20: pobre; 0,21-0,40: débil; 0,41-0,60: moderado; 0,61-0,80: bueno; y 0,81-1,00: muy bueno y coeficiente de correlación intraclase (CCI) para la EVA. Los valores de CCI eran <0,20: nulo acuerdo; entre 0,21 y 0,40 : acuerdo pobre; entre 0,41 y 0,59 : acuerdo justo; entre 0,60 y 0,74: bueno; y entre 0,75 y 1,0: excelente. El diseño de investigación fue descriptivo, de corte transversal.

\section{RESULTADOS \\ Participantes}

El flujograma de participantes (Figura 1) muestra que aceptaron participar 90 niños y sus padres y madres. Debido a que no se presentaron el día de la prueba en el centro, tomaron parte de la

FIGURA 1. Flujograma de participantes

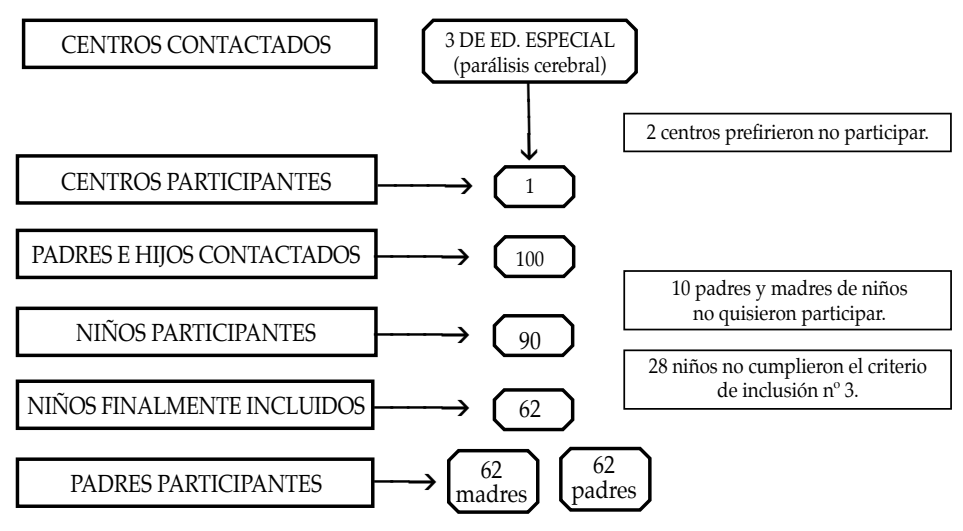


investigación un total de 62 niños con PC, sus padres y madres. En la Tabla 1, se exponen las

TABLA 1. Características de la muestra. $N=62$ niños con parálisis cerebral y sus padres

\begin{tabular}{lc}
\hline & $\mathbf{N}=\mathbf{6 2}$ \\
\hline Edad (en años), M (DT) & $10,09(2,31)$ \\
Niños & $41,2(7,8)$ \\
Padres & $40,7(5,8)$ \\
Madres & \\
Género, n (\%) & $35(56,4)$ \\
Niños & $27(43,6)$ \\
Niñas & \\
País de procedencia & $62(100,0)$ \\
España & $0(0,0)$ \\
Otros & \\
Estatus socioeconómico, n (\%) & $12(19,4)$ \\
Bajo & $38(61,2)$ \\
Medio & $12(19,4)$ \\
Alto & \\
Clasificación funcional de PC & $41(66,1)$ \\
Grado 1 & $21(33,9)$ \\
Grado 2 &
\end{tabular}

M: media; DT: desviación típica; PC: parálisis cerebral. características de la muestra y se puede observar una leve diferencia en cuanto a la distribución por sexo y una predominancia de un estatus socioeconómico medio en el conjunto de la muestra.

\section{Frecuencia de problemas}

En la Tabla 2, se muestra la prevalencia de los problemas reportados por niños y padres y madres. Los resultados muestran 0 valores perdidos.

En líneas generales, se puede observar menor frecuencia de problemas reportados por el padre en las dimensiones del EQ-5D-Y y se acentúa, especialmente, en las dimensiones autocuidado, actividades habituales y ansiedad/depresión. Además, se observa una ligera sobreestimación de la salud en la evaluación que realiza el padre sobre su hijo en la EVA. Sin embargo, la madre reporta un número de problemas superiores al padre, así como una evaluación general de la salud inferior.

\section{Nivel de concordancia}

En la Tabla 3, se presenta el nivel de concordancia entre niño y madre, niño y padre, y madre y padre en población con PC. Se puede observar un bajo acuerdo en todas las comparaciones y en la mayoría de las

TABla 2. Respuestas en las dimensiones del EQ-5D-Y y en su versión Proxy. N: 62

\begin{tabular}{|c|c|c|c|c|c|c|}
\hline \multirow[b]{3}{*}{ Dimensiones EQ-5D-Y } & \multicolumn{4}{|c|}{ Parálisis cerebral } & & \\
\hline & \multicolumn{2}{|c|}{ Niños $(n=62)$} & \multicolumn{2}{|c|}{ Madres (n= 62) } & \multicolumn{2}{|c|}{ Padres $(n=62)$} \\
\hline & $\%$ & n & $\%$ & $\mathbf{n}$ & $\%$ & $\mathbf{n}$ \\
\hline \multicolumn{7}{|l|}{ Movilidad } \\
\hline No tengo problemas para caminar. & 25,8 & 16 & 27,4 & 17 & 33,9 & 21 \\
\hline Tengo algunos problemas para caminar. & 58,1 & 36 & 45,2 & 28 & 54,8 & 34 \\
\hline Tengo muchos problemas para caminar. & 16,1 & 10 & 27,4 & 17 & 11,3 & 7 \\
\hline \multicolumn{7}{|l|}{ Autocuidado } \\
\hline No tengo problemas para vestirme o lavarme solo. & 30,6 & 19 & 54,8 & 34 & 61,3 & 38 \\
\hline Tengo algunos problemas para vestirme o lavarme solo. & 53,2 & 33 & 38,7 & 24 & 35,5 & 22 \\
\hline Tengo muchos problemas para vestirme o lavarme solo. & 16,1 & 10 & 6,5 & 4 & 3,2 & 2 \\
\hline \multicolumn{7}{|l|}{ Actividades habituales } \\
\hline No tengo problemas para realizar mis actividades habituales. & 53,2 & 33 & 58,1 & 36 & 74,2 & 46 \\
\hline Tengo algunos problemas para realizar mis actividades habituales. & 30,6 & 19 & 35,5 & 22 & 25,8 & 16 \\
\hline Tengo muchos problemas para realizar mis actividades habituales. & 16,1 & 10 & 6,5 & 4 & 0,0 & 0 \\
\hline \multicolumn{7}{|l|}{ Dolor/malestar } \\
\hline No tengo dolor o malestar. & 45,2 & 28 & 58,1 & 36 & 69,4 & 43 \\
\hline Tengo algo de dolor o malestar. & 46,8 & 29 & 29,0 & 18 & 22,6 & 14 \\
\hline Tengo mucho dolor o malestar. & 8,1 & 5 & 12,9 & 8 & 8,1 & 5 \\
\hline \multicolumn{7}{|l|}{ Ansiedad/depresión } \\
\hline No me siento preocupado, triste o infeliz. & 53,2 & 33 & 62,9 & 39 & 83,9 & 52 \\
\hline Me siento un poco preocupado, triste o infeliz. & 41,9 & 26 & 33,9 & 21 & 14,5 & 9 \\
\hline Me siento muy preocupado, triste o infeliz. & 4,8 & 3 & 3,2 & 2 & 1,6 & 1 \\
\hline EVA & 70,4 & 12,6 & 76,2 & 14,5 & 80,8 & 14,3 \\
\hline
\end{tabular}

EVA: escala visual analógica; DT: desviación típica.

Valores expresados en porcentajes, excepto la EVA, que está expresada en media (DT). 
dimensiones. Es muy destacable que el nivel de concordancia, siempre que participa en la evaluación de la CVRS el padre, es pobre. Además, el acuerdo entre madre e hijo, a pesar de no tener mucha concordancia, sí que se acerca en las dimensiones de movilidad, con un nivel aceptable $(0,713)$ y actividades habituales, con un nivel de concordancia moderado $(0,436)$.

\section{DISCUSIÓN}

Según la literatura actual, esta es la primera investigación que estudia el grado de acuerdo entre padre e hijo, madre e hijo y padre y madre con el cuestionario de CVRS EQ-5D-Y aplicado en niños y adolescentes que padecen PC. Tres han sido los principales hallazgos de esta investigación. En primer lugar, el padre reporta menos problemas que la madre y que los propios niños en todas las dimensiones. En segundo lugar, existe un alto grado de desacuerdo entre padre y madre en todas las dimensiones. Y, finalmente, es destacable la absoluta discrepancia existente entre padre e hijo y madre e hijo.

Se ha descrito un bajo nivel de acuerdo entre padres e hijos en estudios realizados con el cuestionario Kidscreen sobre niños con $\mathrm{PC}^{17}$ al igual que sucede con el cuestionario PedsQL, en el que se encontró un escaso nivel de concordancia en la dimensión emocional. ${ }^{27}$ Estos hallazgos sostienen nuestra hipótesis sobre la baja concordancia en las funciones psicosociales y físicas, y sus resultados son similares a los encontrados en nuestra investigación. Por tanto, los padres y madres de niños con PC reportan una baja evaluación de CVRS en general y mayores problemas psicológicos que estos, lo que constituye una tendencia en estudios anteriores, como se ha descrito.
Este hecho puede explicarse por la propensión que tienen los niños a enfatizar aspectos positivos sobre la propia enfermedad o adaptación a los cambios. ${ }^{28}$ De esta forma, los padres, de manera conjunta, pueden ser más fiables a la hora de evaluar las capacidades más afectadas de sus hijos, ya que pueden ofrecernos información sobre aquellas dificultades que los niños disimulan. ${ }^{27}$

Además, se encontró una gran discrepancia entre la autoevaluación del niño y la del padre, hecho que contradice anteriores investigaciones que sugerían que el padre era mejor proxy que la madre. ${ }^{29}$ No obstante, dicha investigación se realizó con niños asmáticos, cuyas capacidades no son tan limitantes como las de la población que nos ocupa.

Este hallazgo también se puede observar en nuestro trabajo, en la evaluación de la CVRS de padres, que no tiene tanto grado de acuerdo como con la madre, lo que puede explicarse por el menor tiempo dedicado a la crianza de los hijos por parte del padre. En nuestra investigación, pese al bajo acuerdo entre madre e hijo, este binomio tiene mayor concordancia que padrehijo. Principalmente, son las madres las que realizan la evaluación de la CVRS de sus hijos, ${ }^{30,31}$ por tanto, se cree que basar una evaluación solo en la madre puede desvirtuar, en cierta manera, los resultados. Si bien las madres son las encargadas, habitualmente, del cuidado de los niños, hay ciertos aspectos para tener en cuenta para comprender una evaluación de la CVRS de sus hijos, como el grado de afectación, que va a marcar una tendencia hacia acuerdo o desacuerdo. Este hecho puede hacer que las madres sean más protectoras o que, al contrario, el propio estrés generado por el cuidado de los niños produzca, como se ha visto anteriormente,

TABLA 3. Nivel de acuerdo entre padres e hijos con parálisis cerebral

\begin{tabular}{|c|c|c|c|c|c|c|c|c|c|}
\hline & \multicolumn{3}{|c|}{ Niño-padre $(n=62)$} & \multicolumn{3}{|c|}{ Niño-madre $(n=62)$} & \multicolumn{3}{|c|}{ Padre-madre $(n=62)$} \\
\hline & Kappa & $\begin{array}{l}\text { Valor } \\
\text { de P }\end{array}$ & $\begin{array}{l}\text { Nivel de } \\
\text { acuerdo }\end{array}$ & Kappa & $\begin{array}{l}\text { Valor } \\
\text { de P }\end{array}$ & $\begin{array}{l}\text { Nivel de } \\
\text { acuerdo }\end{array}$ & Kappa & $\begin{array}{l}\text { Valor } \\
\text { de P }\end{array}$ & $\begin{array}{l}\text { Nivel de } \\
\text { acuerdo }\end{array}$ \\
\hline Movilidad & 0,042 & 0,653 & Pobre & 0,713 & $0,000 *$ & Bueno & 0,054 & 0,545 & Pobre \\
\hline Autocuidado & 0,044 & 0,622 & Pobre & 0,057 & 0,536 & Pobre & 0,046 & 0,680 & Pobre \\
\hline Actividades habituales & 0,019 & 0,841 & Pobre & 0,436 & $0,000 *$ & Moderado & 0,182 & 0,096 & Pobre \\
\hline Dolor/malestar & 0,067 & 0,469 & Pobre & 0,128 & 0,183 & Pobre & 0,010 & 0,918 & Pobre \\
\hline Ansiedad/depresión & 0,016 & 0,854 & Pobre & 0,165 & 0,140 & Pobre & 0,030 & 0,765 & Pobre \\
\hline $\operatorname{EVA}(\mathrm{CCI})$ & 0,581 & 0,962 & Justo & 0,389 & 0,029 & Pobre & 0,006 & 0,510 & Nulo \\
\hline
\end{tabular}

* $\mathrm{p}<0,05$.

Niveles de concordancia expresados en kappa de Cohen para las dimensiones del EQ-5D-Y y coeficiente de correlación intraclase (CCI) para la escala visual analógica (EVA). 
una evaluación con tendencia a subestimar la salud de sus hijos.

Esta investigación presenta ciertas limitaciones. En particular, la población objeto de estudio con PC ha sido baja, por lo que no se pueden extraer evidencias. Además, debido a los criterios de inclusión, solo han podido formar parte los afectados con grado 1 y 2 . Otra debilidad es la utilización de un solo cuestionario de CVRS.

Líneas futuras: aumentar la muestra para extraer conclusiones de mayor peso, aplicar un conjunto de cuestionarios más extenso para aportar, así, más información y comparar su aplicabilidad y entre diferentes poblaciones.

\section{CONCLUSIONES}

La principal conclusión que se puede extraer de esta investigación es el alto desacuerdo en la evaluación de la CVRS entre padres e hijos en población con PC a través del cuestionario EQ-5D-Y.

\section{REFERENCIAS}

1. Younossi ZM, Guyatt G. Quality-of-life assessments and chronic liver disease. Am J Gastroenterol 1998;93(7):1037-41.

2. Varni JW, Burwinkle TM, Seid M, et al. The PedsQL 4.0 as a pediatric population health measure: feasibility, reliability, and validity. Ambul Pediatr 2003;3(6):329-41.

3. Ravens-Sieberer U, Gosch A, Rajmil L, etal. KIDSCREEN-52 quality-of-life measure for children and adolescents. Expert Rev Pharmacoecon Outcomes Res 2005;5(3):353-64.

4. Wille N, Badia X, Bonsel G, et al. Development of the EQ5D-Y: a child-friendly version of the EQ-5D. Qual Life Res 2010;19(6):875-86.

5. Ravens-Sieberer U, Erhart M, Rajmil L, et al. Reliability, construct and criterion validity of the KIDSCREEN-10 score: a short measure for children and adolescents' well-being and health-related quality of life. Qual Life Res 2010;19(10):1487-500.

6. Gusi N, Perez-Sousa MA, Gozalo-Delgado M, et al. Validez y fiabilidad de la versión proxy del EQ-5D-Y en español. An Pediatr (Barc) 2014;81(4):212-9.

7. Olivares PR, Perez-Sousa MA, Gozalo-Delgado M, et al. Traducción y adaptación cultural al español de la versión proxy del cuestionario EQ-5D-Y. An Pediatr (Barc) 2013;79(3):157-61.

8. Roizen M, Rodriguez S, Bauer $G$, et al. Initial validation of the Argentinean Spanish version of the PedsQL ${ }^{\mathrm{TM}} 4.0$ Generic Core Scales in children and adolescents with chronic diseases: acceptability and comprehensibility in low-income settings. Health Qual Life Outcomes 2008;6:59.

9. Varni JW, Limbers CA, Burwinkle TM. Parent proxyreport of their children's health-related quality of life: an analysis of 13,878 parents' reliability and validity across age subgroups using the PedsQL ${ }^{\mathrm{TM}} 4.0$ Generic Core Scales. Health Qual Life Outcomes 2007;5:2.

10. Eiser C, Morse R. Quality-of-life measures in chronic diseases of childhood. Health Technol Assess 2001;5(4):1-157.

11. Annett RD, Bender BG, DuHamel TR, et al. Factors influencing parent reports on quality of life for children with asthma. J Asthma 2003;40(5):577-87.

12. Matza LS, Swensen AR, Flood EM, et al. Assessment of health-related quality of life in children: a review of conceptual, methodological, and regulatory issues. Value
Health 2004;7(1):79-92.

13. Le Coq EM, Boeke AJ, Bezemer PD, et al. Which source should we use to measure quality of life in children with asthma: the children themselves or their parents? Qual Life Res 2000;9(6):625-36.

14. Becker A, Hagenberg N, Roessner V, et al. Evaluation of the self-reported SDQ in a clinical setting: Do self-reports tell us more than ratings by adult informants? Eur Child Adolesc Psychiatry 2004;13(Suppl 2):17-24.

15. Van Roy B, Groholt B, Heyerdahl S, et al. Understanding discrepancies in parent-child reporting of emotional and behavioural problems: Effects of relational and sociodemographic factors. BMC Psychiatry 2010;10:56.

16. Russell KM,Hudson M, Long A, et al. Assessment of healthrelated quality of life in children with cancer: Consistency and agreement between parent and child reports. Cancer 2006;106(10):2267-74.

17. White-Koning M, Arnaud C, Dickinson $\mathrm{HO}$, et al. Determinants of child-parent agreement in quality-of-life reports: a European study of children with cerebral palsy. Pediatrics 2007;120(4):e804-14.

18. Sattoe JN, van Staa A, Moll HA, et al. The proxy problem anatomized: Child-parent disagreement in Health Related Quality of Life reports of chronically ill adolescents. Health Qual Life Outcomes 2012;10:10.

19. Upton $P$, Lawford J, Eiser C. Parent-child agreement across child health-related quality of life instruments: a review of the literature. Qual Life Res 2008;17(6):895-913.

20. Lim Y, Velozo C, Bendixen RM. The level of agreement between child self-reports and parent proxy-reports of health-related quality of life in boys with Duchenne muscular dystrophy. Qual Life Res 2014;23(7):1945-52.

21. Ravens-Sieberer U, Wille N, Badia X, et al. Feasibility, reliability, and validity of the EQ-5D-Y: Results from a multinational study. Qual Life Res 2010;19(6):887-97.

22. Wright B, Tindall L, Littlewood E, et al. Computerised cognitive-behavioural therapy for depression in adolescents: feasibility results and 4-month outcomes of a UK randomised controlled trial. BMJ Open 2017;7(1):e012834.

23. Murillo M, Bel J, Pérez J, et al. Health-related quality of life (HRQOL) and its associated factors in children with Type 1 Diabetes Mellitus (T1DM). BMC Pediatr 2017;17(1):16.

24. Bergfors S, Åström M, Burström K, et al. Measuring health-related quality of life with the EQ-5D-Y instrument in children and adolescents with asthma. Acta Paediatr 2015;104(2):167-73.

25. Burström K, Bartonek $\AA$, Broström EW, et al. EQ-5D-Y as a health-related quality of life measure in children and adolescents with functional disability in Sweden: testing feasibility and validity. Acta Paediatr 2014;103(4):426-35.

26. LandisJR, Koch GG. Themeasurement of observer agreement for categorical data. Biometrics 1977;33(1):159-74.

27. Varni JW, Burwinkle TM, Sherman SA, et al. Health-related quality of life of children and adolescents with cerebral palsy: hearing the voices of the children. Dev Med Child Neurol 2005;47(9):592-7.

28. Oeffinger D, Gorton G, Bagley A, etal.Outcome assessments in children with cerebral palsy, Part I: descriptive characteristics of GMFCS Levels I to III. Dev Med Child Neurol 2007;49(3):172-80.

29. Petsios K, Priftis KN, Tsoumakas C, et al. Level of ParentAsthmatic Child Agreement on Health-Related Quality of Life. J Asthma 2011;48(3):286-97.

30. Eiser C, Morse R. A review of measures of quality of life for children with chronic illness. Arch Dis Child 2001;84(3):20511.

31. Jozefiak T, Larsson B, Wichstrom L, et al. Quality of Life as reported by school children and their parents: a crosssectional survey. Health Qual Life Outcomes 2008;6:34. 